\title{
ERRATUM
}

\section{Touch-induced changes in Arabidopsis morphology dependent on gibberellin breakdown}

Maria João Pimenta Lange and Theo Lange

Nature Plants 1, 14025 (2015); published 9 February 2015; corrected 10 February 2015.

In the original version of this Letter, the final row in Fig. $2 \mathrm{~b}$ was incorrectly marked as significant. This error has been corrected 10 February 2015. 